\title{
Risk factors, aetiology, therapy and outcome in 123 episodes of breakthrough bacteraemia and fungaemia during antimicrobial prophylaxis and therapy in cancer patients
}

\author{
S. SPANIK, J. TRUPL $\ddagger$ A. KUNOVA*, L. DRGONA†, T. SALEK, J. MARDIAK, E. KUKUCKOVA*, \\ M. STUDENA*, P. PICHNA†, E. ORAVCOVA, E. GREY, P. KOREN*, J. SVEC§, J. LACKA, \\ J. SUFLIARSKY and $V$. KRCMERY \\ St Elizabeth National Cancer Institute, Bratislava, ${ }^{*}$ Department of Medicine, University of Trnava, + Department \\ of Chemotherapy, Postgraduate Medical School, $\$$ Department of Microbiology, Hematology and Medicine, \\ National Cancer Institute and §Department of Oncology, Medical School, Commenius University, Bratislava, \\ Slovak Republic
}

\begin{abstract}
One hundred and twenty-three breakthrough bacteraemias (BB) were defined during a 5-year period in a National Cancer Centre, among 9986 admissions and a total of 979 bacteraemic episodes analysed. Of 123 bacteraemias in 103 patients, 77 were polymicrobial and 116 of the 323 organisms isolated were resistant to currently administered antimicrobial agents. Sixty-seven of the bacteraemic episodes were catheter-associated, as confirmed by the isolation of the same organisms from both blood and catheter tip. The strains isolated most frequently were coagulase-negative staphylococci $(30.5 \%)$, corynebacteria $(10 \%)$, Pseudomonas aeruginosa $(10 \%)$, Enterococcus faecalis $(9 \%)$ and viridans streptococci $(8.5 \%)$. Gram-positive aerobes accounted for two-thirds of all micro-organisms isolated during breakthrough bacteraemic and fungaemic episodes. Polymicrobial episodes were associated more frequently with vascular catheters and neutropenia, and had a less favourable outcome than monomicrobial infections. Relapse was associated more frequently with catheter-related episodes, but the overall mortality rate was similar and independent of catheter insertion. Breakthrough bacteraemic and fungaemic episodes were associated more frequently with acute leukaemia. Catheter removal, as an independent variable, and modification of antimicrobial therapy were essential for better outcome.
\end{abstract}

\section{Introduction}

The incidence of bacteraemia and fungaemia has increased steadily during the past decade, despite the use of broad-spectrum empiric therapy with bactericidal drugs. Recent EORTC studies showed that bacteraemic episodes were associated more frequently with therapy failure and a worse outcome was observed in polymicrobial and intravascular catheter-associated bacteraemia and fungaemia [1-7]. In large studies on prevention and therapy of febrile neutropenia, breakthrough bacteraemias (BB) are increasingly common [4-8]. Several outbreaks of BB and fungaemia have

Received 10 Oct. 1995; revised version accepted 20 Oct. 1996.

Corresponding author: Professor Dr V. Krcmery. been reported from cancer centres worldwide either due to quinolone-resistant Enterobacteriaceae [9-11], vancomycin-resistant enterococci [12], penicillin-resistant viridans streptococci [13], meropenem-resistant Pseudomonas spp. [14] or to azole-resistant Candida spp. $[15,16]$.

Mortality following gram-positive bacteraemia, with the exception of viridans streptococci, is low [3-7]. Once quinolones were introduced into the prophylactic regimen, the incidence and mortality following gramnegative bacteraemia decreased, although fungaemia is still associated with $25-40 \%$ mortality.

Apart from catheters (as a source of persisting bacteraemia), infection with resistant strains and neutropenia are recognised risk factors for breakthrough bacteraemia and fungaemia in cancer patients. 
The aim of this study was to investigate the incidence, aetiology, risk factors and outcome of BB over 5 years in the National Cancer Centre of the Slovak Republic.

\section{Patients and methods}

A total of 979 bacteraemic and fungaemic episodes among 9986 admissions between 1 Jan. 1990 and 31 Dec. 1994 (5 years) at the 500-bed National Cancer Institute of the Slovak Republic were analysed to assess breakthrough bacteraemic and fungaemic episodes. There were 123 episodes of BB in 103 patients, with 323 isolates from blood cultures. BB was defined by a positive blood culture drawn through a peripheral vein, following symptoms, during antibiotic therapy or prophylaxis with absorbable drugs. Patients with haematological malignancies and neutropenia $<500$ / $\mathrm{ml}$ routinely receive ofloxacin plus fluconazole or itraconazole orally. Blood cultures were drawn from a peripheral vein and the catheter, only on suspicion of a vascular catheter-associated BB or fungaemic episode. The catheter was removed routinely when positive blood cultures or polymicrobial BB appeared, except in the case of five patients with thrombocytopenia $<20000 / \mathrm{ml}$. Routine blood culture was performed by the BACTEC system (Becton Dickinson). Antibiotic susceptibility testing was performed according to the NCCLS methods.

Catheter-associated BB was defined when the same organism(s) were isolated from blood culture and catheter tip. Polymicrobial BB was defined as the isolation of two or more different species from blood culture.

Neutropenia was defined by a neutrophil count of persistently $<500 / \mathrm{ml}$ at the time a blood culture was taken.

Cure was accepted if patients' signs and symptoms disappeared. Failures included relapse, or death during or because of bacteraemia or fungaemia. Relapse was defined by a second episode within 10 days during one hospitalisation. Death during BB was not always clearly associated with sepsis. On the other hand, where death occurred $\mathrm{BB}$ or within 5 days of an episode and followed clinically documented sepsis it was deemed a treatment failure (death due to bacteraemia or fungaemia).

Risk factors associated with $\mathrm{BB}$, such as underlying disease, neutropenia, presence of central or peripheral venous catheter or presence of resistant isolates in blood culture were recorded. Aetiology, therapy and outcome of patients with BB were also recorded.

For statistical analysis the $x^{2}$ test was used to compare different subgroups and $\mathrm{p}<0.05$ was considered a significant difference.

\section{Results and discussion}

The incidence and distribution of $\mathrm{BB}$ are outlined in Table 1: among 9986 admissions, there were 979 bacteraemic and fungaemic episodes and $123(13 \%)$ were breakthrough (BB). Of the latter, approximately one-half were vascular catheter-associated and twothirds were polymicrobial.

Patient details, underlying disease, isolated microorganism, antibiotic therapy, onset of bacteraemia, risk factors (e.g., catheter, neutropenia, microbial resistance), therapy and outcome are shown in Tables 2-4, separately for monomicrobial and polymicrobial, and vascular catheter-associated BB. Tables $2-4$ show the assessment of risk factors for catheter-associated BB. Study of the risk of vascular catheter-associated and non-vascular catheter-associated bacteraemia indicated that there was no difference between the groups in the appearance of BB during therapy or prophylaxis $(80.6$ and $78 \%$ BB appeared during therapy and 19.4 and $25 \%$ during prophylaxis with ofloxacin, respectively). There was a higher incidence of neutropenia or resistant organisms or both in non-vascular catheterassociated BB when compared with catheter-related BB. Other risk factors such as neutropenia or identification of resistant strains from blood cultures, or both, were more frequently associated with the group of vascular catheter-associated BB.

There were similar cure rates $(51.2 \%$ in all patients with BB) with no significant difference between catheter- and non-catheter-related $\mathrm{BB}$, although there was a higher relapse rate while a vascular catheter was in situ $(14.9 \%$ versus $7.1 \%, \mathrm{p}<0.03)$. Surprisingly, death due to bacteraemia or septic shock was more frequently registered in patients without catheters. When analysing therapy and action taken in BB, with and without vascular catheter, the failure rate was $100 \%$ in those where no action or therapy was taken. There was no difference in outcome, once the catheter was removed, whether or not antibiotic therapy was modified. This finding suggests that catheter removal is essential for cure. Change of antimicrobial agent alone, in the group of patients

Table 1. Distribution of breakthrough bacteraemic episodes among 979 bacteraemic and fungaemic episodes within 5 years among 9986 admissions

\begin{tabular}{lc}
\hline Criteria & $\begin{array}{c}\text { Number of } \\
\text { patients }\end{array}$ \\
\hline Admissions (1.1.1989-31.12.1994) & 9986 \\
Patients with bacteraemia & 443 \\
Bacteraemic episodes (BE) & 979 \\
Breakthrough bacteraemia episodes (BB) & 123 \\
Strains isolated from BB & 323 \\
Vascular catheter-associated BB & $67(54.5 \%)$ \\
Non-vascular catheter-associated BB & $56(45.5 \%)$ \\
Monomicrobial BB & $46(37.4 \%)$ \\
Polymicrobial BB & $77(62.6 \%)$ \\
\hline
\end{tabular}


Table 2. Breakthrough bacteraemia (BB) in 123 patients: its relationship with other risk factors and catheter association

\begin{tabular}{|c|c|c|c|c|}
\hline Criteria & Total & $\begin{array}{l}\text { Catheter- } \\
\text { associated } \\
\text { bacteraemia }\end{array}$ & $\begin{array}{l}\text { Non-catheter- } \\
\text { associated } \\
\text { bacteraemia }\end{array}$ & $\mathrm{p}$ value \\
\hline Number of patients & 123 & 67 & 56 & \\
\hline BB during therapy & $96(78 \%)$ & $54(80.6 \%)$ & $42(75 \%)$ & NS \\
\hline BB during prophylaxis & $27(22 \%)$ & $13(19.4 \%)$ & $14(25 \%)$ & NS \\
\hline \multicolumn{5}{|l|}{ Underlying disease } \\
\hline Acute leukaemia & $67(54.5)$ & $45(67.2)$ & $22(39.3)$ & 0.02 \\
\hline Other haematological disorders & $22(17.9)$ & $11(16.4)$ & $11(19.6)$ & NS \\
\hline Solid tumours & $34(27.6)$ & $11(16.4)$ & $23(41.1)$ & 0.02 \\
\hline \multicolumn{5}{|l|}{ Risk factors } \\
\hline Catheter only & $9(7.3)$ & $9(13.4)$ & 0 & 0.001 \\
\hline Neutropenia only & $7(5.7)$ & 0 & $7(12.5)$ & 0.001 \\
\hline Resistant organism only & $14(11.4)$ & 0 & $14(25)$ & 0.001 \\
\hline Neutropenia + resistance & $35(28.5)$ & 0 & $35(62.5)$ & 0.001 \\
\hline Catheter + neutropenia & $30(24.4)$ & $30(44.8)$ & 0 & 0.001 \\
\hline Catheter + resistance & $9(7.3)$ & $9(13.4)$ & 0 & 0.001 \\
\hline Catheter + resistance + neutropenia & $19(15.4)$ & $19(28.4)$ & 0 & 0.001 \\
\hline \multicolumn{5}{|l|}{ Therapy } \\
\hline Antibiotic change/failures & $47 / 20(42.5)$ & 0 & $47 / 20(42.5)$ & 0.001 \\
\hline Catheter extracted + antibiotic not changed/failures & $9 / 2(22.2)$ & $9 / 2(22.2)$ & 0 & 0.001 \\
\hline Catheter extracted + antibiotic changed/failures & $58 / 29(50)$ & $58 / 29(50)$ & 0 & 0.001 \\
\hline No therapy/failures & $9 / 9(100)$ & 0 & $9 / 9(100)$ & 0.001 \\
\hline \multicolumn{5}{|l|}{ Aetiology } \\
\hline $\mathrm{G}^{+}$ & $55(44.8)$ & $23(34.3)$ & $32(57.1)$ & 0.04 \\
\hline $\mathrm{G}^{-}$ & $22(17.8)$ & $10(14.9)$ & $12(21.4)$ & NS \\
\hline Mixed $\mathrm{G}^{+}$and $\mathrm{G}^{-}$(fungi) & $46(37.4)$ & $34(50.8)$ & $12(21.4)$ & 0.02 \\
\hline \multicolumn{5}{|l|}{ Outcome } \\
\hline Cured & $63(51.2)$ & $36(53.8)$ & $27(48.2)$ & NS \\
\hline \multirow{3}{*}{$\begin{aligned} \text { Failures } & \text { - cured but relapsed } \\
& \text { - death with bacteraemia } \\
& \text { - death due to bacteraemia (septi }\end{aligned}$} & $14(11.4)$ & $10(14.9)$ & $4(7.1)$ & 0.03 \\
\hline & $18.1(14.6)$ & $9(13.4)$ & $9(16.1)$ & NS \\
\hline & $28(22.8)$ & $12(17.9)$ & $16(28.6)$ & 0.03 \\
\hline
\end{tabular}

$\mathrm{G}^{+}$, gram-positive bacteria; $\mathrm{G}^{-}$, gram-negative bacteria.

Table 3. Breakthrough bacteraemia (BB) in 123 patients: risk factors associated with monomicrobial and polymicrobial $\mathrm{BB}$

\begin{tabular}{|c|c|c|c|}
\hline Criteria & $\begin{array}{c}\text { Monomicrobial } \\
(\%)\end{array}$ & $\begin{array}{c}\text { Polymicrobial } \\
(\%)\end{array}$ & $\mathrm{p}$ value \\
\hline $\mathrm{BB}$ occurring during therapy for $\mathrm{BB}$ & $36(78.3)$ & $60(77.9)$ & NS \\
\hline BB occurring during prophylaxis & $10(21.7)$ & $17(22.1)$ & NS \\
\hline \multicolumn{4}{|l|}{ Underlying disease } \\
\hline Acute leukaemia & $20(43.4)$ & $47(61)$ & 0.05 \\
\hline Other haematological disorders & $10(21.8)$ & $12(15.6)$ & 0.05 \\
\hline Solid tumours & $16(34.8)$ & $18(23.4)$ & 0.03 \\
\hline \multicolumn{4}{|l|}{ Risk factors } \\
\hline Catheter only & $5(10.9)$ & $4(5.2)$ & 0.03 \\
\hline Neutropenia only & $4(8.7)$ & $3(3.9)$ & 0.03 \\
\hline Resistant organism only & $7(15.2)$ & $7(9.1)$ & NS \\
\hline Neutropenia + resistance & $15(32.6)$ & $20(26)$ & NS \\
\hline Catheter + neutropenia & $8(17.4)$ & $22(28.6)$ & 0.03 \\
\hline Catheter + resistance & $3(6.5)$ & $6(7.8)$ & NS \\
\hline Catheter + resistance + neutropenia & $4(8.7)$ & $15(19.5)$ & 0.03 \\
\hline \multicolumn{4}{|l|}{ Therapy } \\
\hline Antibiotic change (cure/failure) & $22 / 9$ & $25 / 11$ & - \\
\hline Catheter extracted (cure/failure) & $9 / 2$ & $0 / 0$ & - \\
\hline Catheter extracted + antibiotic change (cure/failure) & $11 / 5$ & $47 / 24$ & - \\
\hline No therapy (cure/failure) & $4 / 4$ & $5 / 5$ & - \\
\hline \multicolumn{4}{|l|}{ Aetiology } \\
\hline $\mathrm{G}^{+}$ & $27(58.7)$ & $28(36.4)$ & NS \\
\hline $\mathrm{G}^{-}$ & $19(41.3)$ & $3(3.9)$ & 0.005 \\
\hline Mixed $\mathrm{G}^{+}$and $\mathrm{G}^{-}$(fungi) & $0 / 0$ & $46(59.7)$ & 0.0001 \\
\hline \multicolumn{4}{|l|}{ Outcome } \\
\hline Cured & $26(56.3)$ & $37(48)$ & NS \\
\hline Failures - cured but relapsed and died & $6(13.1)$ & $8(10.4)$ & NS \\
\hline- death with bacteraemia & $6(13.1)$ & $12(15.6)$ & NS \\
\hline - death due to bacteraemia (septic shock) & $8(17.5)$ & $20(26)$ & NS \\
\hline Number of patients (total $=100 \%$ ) & 46 & 77 & \\
\hline
\end{tabular}

$\mathrm{G}^{+}$, gram-positive bacteria; $\mathrm{G}^{-}$, gram-negative bacteria. 
Table 4. Breakthrough bacteraemia (BB) in 123 patients: risk factors related to outcome

\begin{tabular}{|c|c|c|c|c|c|c|}
\hline & Total & $\begin{array}{c}\text { Cured patients } \\
(\%)\end{array}$ & $\begin{array}{l}\text { Failed (died or } \\
\text { relapsed) }(\%)\end{array}$ & Died & $\begin{array}{l}\text { Cured but } \\
\text { relapsed }\end{array}$ & \\
\hline \multicolumn{7}{|l|}{ Time of $B B$} \\
\hline $\mathrm{BB}$ occurring during therapy for $\mathrm{BB}$ & 96 & $49(77.8)$ & $47(78.3)$ & 39 & 8 & NS \\
\hline BB occurring during prophylaxis & 27 & $14(22.2)$ & $13(21.7)$ & 7 & 6 & NS \\
\hline \multicolumn{7}{|l|}{ Underlying disease } \\
\hline Acute leukaemia & 67 & $37(58.9)$ & $30(50)$ & 19 & 11 & NS \\
\hline Other haematological disorders & 22 & $8(12.7)$ & $14(23.3)$ & 12 & 2 & NS \\
\hline Solid tumours & 34 & $18(28.6)$ & $16(26.6)$ & 15 & 1 & NS \\
\hline \multicolumn{7}{|l|}{ Risk factors } \\
\hline Catheter-associated & 67 & $36(57.1)$ & $31(51.7)$ & 21 & 10 & NS \\
\hline Non-catheter-associated & 56 & $27(42.8)$ & $29(48.3)$ & 25 & 4 & NS \\
\hline Catheter only & 9 & $8(12.7)$ & $1(1.7)$ & 1 & 0 & 0.01 \\
\hline Neutropenia only & 7 & $2(3.2)$ & $5(8.3)$ & 5 & 0 & 0.01 \\
\hline Resistant organism only & 14 & $7(11.1)$ & $7(11.7)$ & 7 & 0 & NS \\
\hline Neutropenia plus resistant organism & 35 & $17(27)$ & $18(30)$ & 14 & 4 & NS \\
\hline Catheter plus neutropenia & 30 & $15(23.8)$ & $15(25)$ & 11 & 4 & NS \\
\hline Catheter plus resistance & 9 & $5(7.9)$ & $4(6.7)$ & 3 & 1 & NS \\
\hline Catheter plus resistance plus neutropenia & 19 & $9(14.3)$ & $10(16.7)$ & 5 & 5 & NS \\
\hline \multicolumn{7}{|l|}{ Aetiology } \\
\hline $\mathrm{G}^{+}$(polymicrobial) & 28 & $15(23.8)$ & $13(21.7)$ & 8 & 5 & NS \\
\hline $\mathrm{G}^{-}$(polymicrobial) & 3 & 0 & $3(5)$ & 2 & 1 & 0.01 \\
\hline Mixed $\mathrm{G}^{+}$and $\mathrm{G}^{-}$(polymicrobial) & 46 & $22(34.9)$ & $24(40)$ & 18 & 6 & NS \\
\hline $\mathrm{G}^{+}$(monomicrobial) & 27 & $13(20.1)$ & $14(23.3)$ & 10 & 4 & NS \\
\hline $\mathrm{G}^{-\prime}$ (monomicrobial) & 19 & $13(20.1)$ & $6(10)$ & 4 & 2 & NS \\
\hline Number of patients (total $=100 \%$ ) & 123 & 63 & 60 & 42 & 18 & \\
\hline \multicolumn{7}{|l|}{ Therapy/action } \\
\hline Antibiotic change & 47 & $27(42.8)$ & $20(33.3)$ & 16 & 4 & NS \\
\hline Catheter extraction antibiotic not changed & 9 & $7(11.1)$ & $2(3.3)$ & 1 & 1 & 0.01 \\
\hline Catheter extracted plus antibiotic changed & 58 & $29(46.1)$ & $29(48.3)$ & 20 & 9 & NS \\
\hline No therapy/no action & 9 & 0 & $9(15)$ & 9 & 0 & 0.001 \\
\hline
\end{tabular}

$\mathrm{G}^{+}$, gram-positive bacteria; $\mathrm{G}^{-}$, gram-negative bacteria.

without vascular catheters, was as effective as catheter removal plus antibiotic change in patients with a vascular catheter.

Table 3 shows the relationship between risk factors and outcome in monomicrobial BB and polymicrobial $\mathrm{BB}$; no difference between the groups was observed when BB occurred during therapy or prophylaxis. The majority of monomicrobial and polymicrobial (78.3 and $77.9 \%$ ) $\mathrm{BB}$ appeared during therapy. No patients had both polymicrobial plus monomicrobial $\mathrm{BB}$ on different occasions. Polymicrobial BB occurred rather more frequently in patients with acute leukaemia (AL) (61.0\% patients with polymicrobial $\mathrm{BB}$ had $\mathrm{AL}$ while $43.4 \%$ with monomicrobial $\mathrm{BB}$ had $\mathrm{AL}$ ). A statistically significant risk for polymicrobial BB was the presence of neutropenia plus catheter or isolation of resistant strains, or both $(19.5 \%$ polymicrobial versus $8.7 \%$ monomicrobial BB, $p<0.03)$. Failure was independent of therapy and action taken except for catheter removal (catheters were removed in only nine cases of monomicrobial $\mathrm{BB}$, and the relapse rate was only $22.2 \%$ compared with a total of $43-52 \%$ failures). Change of antimicrobial agents only in both monomicrobial and polymicrobial $\mathrm{BB}$ was equally unsuccessful (43 versus 44\%, not significant).

Monomicrobial BB was more frequently associated with gram-positive $(51.7 \%)$ than gram-negative organisms $(41.3 \%)$, but in polymicrobial $\mathrm{BB} 36.4 \%$ were due to two or more gram-positive species, only $3.9 \%$ were due to two gram-negative species and $59.7 \%$ to mixed aetiology.

There was no statisticallly significant difference in rates of cure, relapse and death, between monomicrobial polymicrobial BB. This was an unexpected finding, because polymicrobial bacteraemia in similar studies has been associated more often with failure $[5-8,16]$.

Table 4 shows the association between outcome and various risk factors: there was no significant difference in outcome if $\mathrm{BB}$ occurred during prophylaxis or therapy if the patient had AL or other malignancy (haematological or solid tumour). More patients failed in the subgroup of polymicrobial BB than monomicrobial $\mathrm{BB}$ when $\mathrm{BB}$ was caused by a resistant strain $(p<0.04)$ or a gram-negative organism. Higher cure rates were observed in patients with $\mathrm{BB}$ after catheter extraction, whether or not antimicrobial agents were changed, as already observed in the analyses in Tables 2 and 3 . This confirms the importance of catheter removal for a successful outcome.

Table 5 shows the association between the antibiotic resistance of the organisms and the antibiotic regimen during which BB appeared. During ofloxacin prophylaxis, 11 vascular catheter-associated and nine noncatheter-associated BB were noted, most frequently caused by coagulase-negative staphylococci (CNS), 


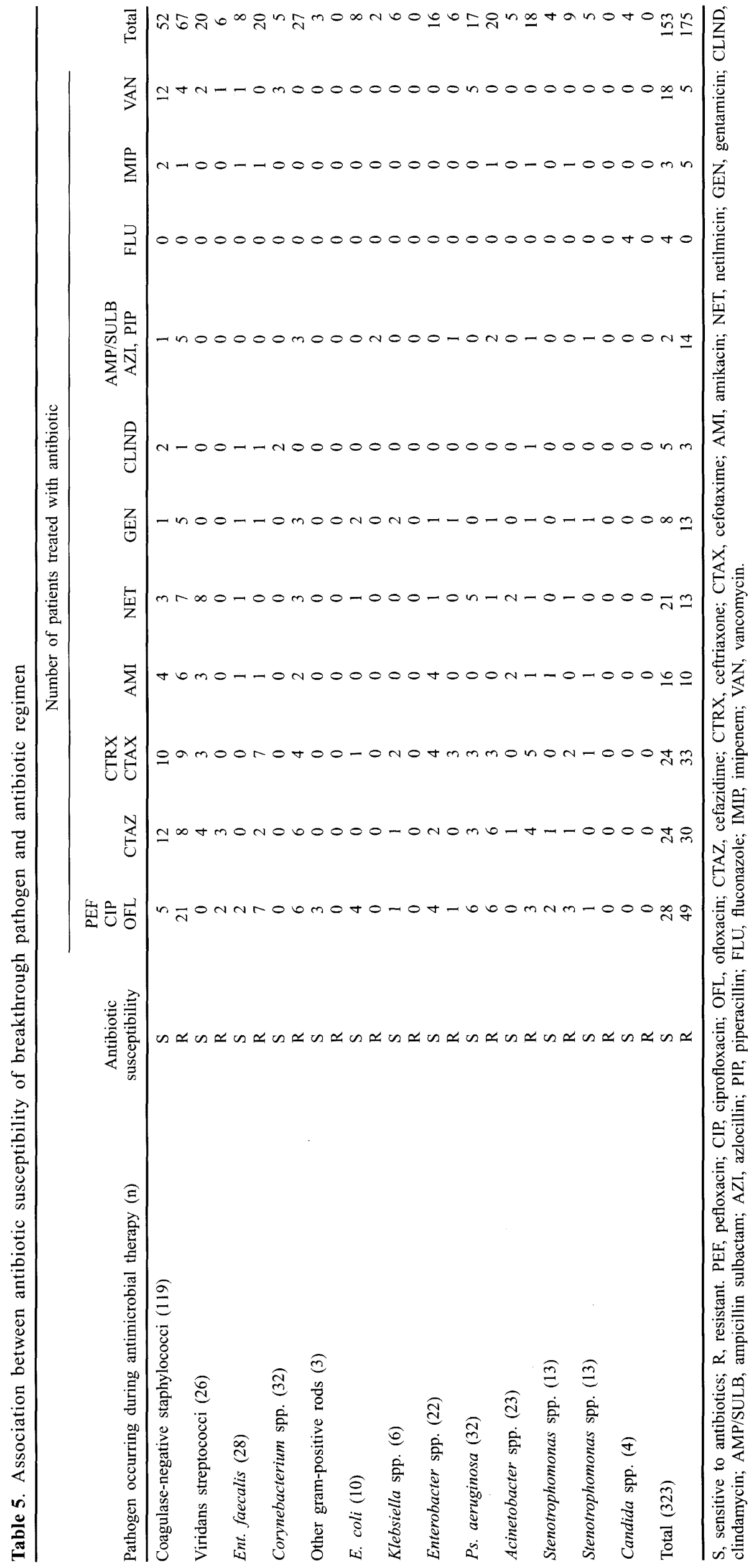


Enterococcus faecalis, viridans streptococci, or PS. aeruginosa.

During therapy with quinolones (parental ofloxacin, ciprofloxacin or perfloxacin), the most common breakthrough organisms were: CNS (five quinolone sensitive, 21 resistant strains), Ps. aeruginosa (six sensitive, six resistant), Ent. faecalis (two sensitive, seven resistant), Stenotrophomonas maltophilia (two sensitive, three resistant), Corynebacterium spp. and others (Table 5).

Despite therapy with ceftazidime (CTAZ), 44 organisms were isolated: 20 were CNS, seven viridans streptococci, nine Ps. aeruginosa (six CTAZ-resistant, three CTAZ-sensitive) and six Corynebacterium spp.

During therapy with cefotaxime (CTAX) or ceftriaxone (CTRX), the most frequently isolated strains among BB were CNS (19 strains, nine resistant), Ent. faecalis (seven, all resistant), Enterobacter spp. (seven strains, three CTAX- or CTRX-resistant), Ps. aeruginosa (six, three resistant).

During therapy with aminoglysides 81 organisms were isolated: amikacin 26, netilmicin 34 and gentamicin 21 and the most commonly isolated organisms were CNS (26 strains), viridans streptococci (11), Ps. aeruginosa, Acinetobacter and surprisingly also Enterobacter spp. (seven each).

During therapy with penicillins (ampicillin sulbactam, azlocillin, piperacillin) BB occurred in only 16 cases, mostly due to CNS. Of eight strains breaking through therapy with imipenem, five were resistant to the antibiotic (one Acinetobacter sp., one Sten. maltophilia, one Ps. aeruginosa, one Ent. faecalis, one CNS). Surprisingly, $23 \mathrm{BB}$ due to gram-positive bacteria also occurred during vancomycin (VAN) therapy, and five were VAN-resistant $(S$. epidermidis four strains, one viridans streptococcus), which is an unusual finding; $S$. epidermidis strains had MICs of VAN of $8-32 \mathrm{mg}$ / $\mathrm{L}$ and the viridans streptococcus $16 \mathrm{mg} / \mathrm{L}$. However, no $\mathrm{BB}$ due to VAN-resistant Ent. faecalis was observed, unlike other cancer centres [12]

CNS were the most frequent cause of $\mathrm{BB}$ and appeared in $116(31.5 \%)$ of 322 blood cultures. Also common causes of $\mathrm{BB}$ were Ps. aeruginosa and Corynebacterium spp., 32 episodes each (9.9\%) and Ent. faecalis (28 episodes).

In similar studies of $\mathrm{BB}$ [2-7], the most frequent risk factors were venous or urinary catheters along with persisting bacteraemia, age, diabetes mellitus, neoplasia and resistance of strains isolated during bacteraemia. In contrast to those studies, no clear benefit was observed in the immunosuppressed population following early change of therapy. In all those studies, if bacteraemia and fungaemia appeared catheter extraction (if present) was independently beneficial for outcome. Optimal therapy duration for episodes is recommended to be up to 14 days $[18-20]$.

Although VAN-resistance in viridans streptococci and CNS is rare in comparison to Ent. faecium, four resistant CNS and one VAN-resistant viridans streptococcus strain were observed, which has not been reported in other studies. This may be due to increased use of VAN in our centre, when use of VAN in 1994 was eight times that used in 1990.

\section{References}

1. EORTC Antimicrobial Therapy Group. Prospective comparison of three antibiotic regimens for empirical therapy of suspected infection in febrile granulocytopenia patients. I Infect Dis 1978; 137: 22--29

2. EORTC International Antimicrobial Therapy Cooperative Group. Ceftazidime combined with a short or long course of amikacin for empirical therapy of gram-negative bacteremia in cancer patients with granulocytopenia. N Engl J Med 1987; 317: $1692-1698$

3. EORTC International Antimicrobial Therapy Cooperative Group and the National Cancer Institute of Canada - Clinical Trials Group. Vancomycin added to empirical combination antibiotic therapy for fever in granulocytopenic cancer patients. $J$ Infect Dis 1991; 163: 951-958.

4. Meunier F, Zinner SH, Gaya $\mathrm{H}$ and the EORTC Antimicrobial Therapy Group. Prospective randomized evaluation of ciprofloxacin versus piperacillin plus amikacin for empiric antibiotic therapy of febrile granulocytopenic cancer patients with lymphomas and solid tumors. Antimicrob Agents Chemother 1991; 35: 873-878.

5. The International Antimicrobial Therapy Cooperative Group of the European Organization for Research and Treatment of Cancer. Efficacy and toxicity of single daily doses of amikacin and cetriaxone versus multiple daily doses of amikacin and ceftazidime for infection in patients with cancer and granulocytopenia. Ann Intern Med 1993; 119: 584-593.

6. EORTC International Antimicrobial Therapy Cooperative Group. A prospective double blind study of pefloxacin plus penicillin or placebo for prophylaxis of bacterial infections in granulocytopenic cancer patients. 32nd ICAAC, Anaheim. 1992. Abstract no. 1692 .

7. Gaya $\mathrm{H}$, and the International Antimicrobial Therapy Cooperative Group. Piperacillin/tazobactam plus amikacin versus ceftazidime plus amikacin as empirical therapy for fever in patients with granulocytopenia. 33rd ICAAC, New Orleans. 1993. Abstract no. 645 .

8. International Antimicrobial Therapy Cooperative Group of the EORTC. A randomized study on meropenem versus ceftazidim plus amikacin in febrile granulocytopenic patients. Abstracts, 34th ICAAC, Orlando. 1994. Abstract no. 1193.

9. Kern W, Andriof E, Oethinger M, Kern P, Hacker J, Marre R. Emergence of fluoroquinolone-resistant Escherichia coli at a cancer center. Antimicrob Agents Chemother 1994; 38: $681-687$.

10. Cometta A, Calandra T, Bille T, Galazzo J, Giddey M, Glauser M. Quinolone resistant Escherichia coli bacteraemias in neutropenic cancer patients. Proceedings of the IHS Symposium, Davos, 1994. Abstract no. 84

11. Carratala J, Fernandez-Swilla A, Tubau F, Callis M, Gudiol F. Emergence of quinolone resistant Escherichia coli bacteremia in neutropenic patients with cancer who have received prophylactic norfloxacin. Clin Infect Dis 1995; 20: 557-560.

12. Noskin GA, Peterson LR, Warren JR. Enterococcus faecium and Enterococcus faecalis bacteraemia: acquisition and outcome. Clin Infect Dis 1995; 20: 296-301.

13. Bochud P, Eggimann P, Calandra T, Van Melle G, Saghafi L, Franciol P. Bacteremia due to viridans streptococcus in neutropenic patients with cancer: clinical spectrum and risk factors. Clin Infect Dis 1994; 18: 25.

14. Krčméry V, Trupl J. Nosocomial outbreak of meropenem 
resistant Pseudomonas aeruginosa infections in a cancer centre. J Hosp Infect 1994; 26: 69-71

15. Wingard JR, Merz WG, Rinaldi MG, Johnson TR, Karp JE, Sarah R. Increase in Candida krusei infection among patients with bone marrow transplantation and neutropenia treated prophylactically with fluconazole. $N$ Engl $J$ Med 1991; 325: $1274-1277$.

16. Viscoli C. Surveillance of fungaemias in cancer centres EORTC study. Abstracts, 6th BICON Annual Meeting Bratislava. 1995. Abstract no. 33; 19.-23.3.

17. Chow J, Fine M, Shlaes DM et al. Enterobacter bacteraemia: clinical features and emergence of antibiotic resistance during therapy. Ann Infect Med 1991; 115: 585-590.

18. Rosenthal EJK. [Epidemiologie von Septikämie-Erregen. Blutkulturstudie der Paul-Ehrlich-Gesellschaft für Chemotherapie e.V.] Dtsch Med Wochenschr 1993; 118: 1269-1275.

19. Lecciones J, Lee J, Navarro E et al. Vascular catheterassociated fungemia in patients with cancer: analysis of 155 episodes. Clin Infect Dis 1992; 16: 875.

20. Tilley G, Roberts F. Bacteremia with Acinetobacter species: risk factors and prognosis in different clinical settings. Clin Infect Dis 1994; 18: 896-900. 